\title{
Fertilización orgánica contra convencional en el rendimiento, atributos morfológicos y calidad de fruto de tomate uva en un sistema de subirrigación no recirculante Organic versus conventional fertilization on yield, morphological attributes and fruit quality of grape tomato in a non-recirculating subirrigation system
}

\author{
José Antonio González-Fuentes ${ }^{1}{ }^{(1)}$, Carlos Javier Lozano-Cavazos ${ }^{2:}{ }^{(1)}$, Pablo Preciado-Rangel ${ }^{3}$ (1), \\ Enrique Troyo-Diéguez ${ }^{4}$ (i) Alfonso Rojas-Duarte ${ }^{1}$ (i) y Juan Carlos Rodríguez-Ortiz $^{5}$ (i)
}

\footnotetext{
${ }^{1}$ Departamento de Horticultura, ${ }^{2}$ Departamento de Fitomejoramiento. Universidad Autónoma Agraria "Antonio Narro". Calzada Antonio Narro 1923, Buenavista. 25315 Saltillo, Coahuila, México.

¥ Autor para correspondencia (carlos.lozano@uaaan.edu.mx)

${ }^{3}$ Tecnológico Nacional de México/Instituto Tecnológico de Torreón. Carretera Torreón - San Pedro de las Colonias km 7.5, Ejido Ana. 27170 Torreón, Coahuila, México.

${ }^{4}$ Centro de Investigaciones Biológicas del Noroeste SC, Programa de Agricultura en Zonas Áridas. Av. Instituto Politécnico Nacional 195, Col. Playa Palo de Santa Rita Sur. 23096 La Paz, B.C.S., México.

${ }^{5}$ Facultad de Agronomía y Veterinaria. Universidad Autónoma de San Luis Potosí. Carretera San Luis - Matehuala km 14.5, Ejido Palma de la Cruz. 78321 Soledad de Graciano Sánchez, S.L.P., México.
}

\section{RESUMEN}

En el ámbito de la agricultura sostenible, el uso de fertilizantes orgánicos es una opción viable ante el impacto ambiental causado por los fertilizantes convencionales. El objetivo de este trabajo fue evaluar el efecto de abonos orgánicos como fuente de $\mathrm{N}$ sobre el crecimiento, rendimiento, calidad y atributos morfológicos en frutos de tomates uva. Se evaluaron siete tratamientos de fertilización, los cuales consistieron de una solución nutritiva inorgánica (solución Steiner) y diferentes mezclas de varios fertilizantes nitrogenados de origen animal certificados por OMRI a una concentración del 100 y 120\%. Los resultados mostraron que los tratamientos orgánicos T5 y T6 presentaron similar crecimiento que el tratamiento convencional (T7). El índice SPAD fue $13.8 \%$ mayor en los tratamientos orgánicos respecto al tratamiento inorgánico T7. En los parámetros de producción de fruto, el tratamiento orgánico T5 asemejó al tratamiento convencional en el número de frutos por racimo, pero en peso fresco de fruto, rendimiento y diámetro longitudinal el tratamiento convencional superó a los tratamientos orgánicos por un 21, 31.6 y 5.8\%; mientras que, el diámetro transversal fue similar en los tratamientos T1 y T7. La firmeza de fruto fue mayor en los tratamientos orgánicos T1 y T4, superando al tratamiento inorgánico por 10.3 y $6 \%$, respectivamente. Los sólidos solubles totales fueron mayores en el tratamiento orgánico $\mathrm{T} 4$, superando al tratamiento inorgánico $\mathrm{T} 7$ por un $18 \%$. El tratamiento inorgánico $\mathrm{T} 7$ presentó mayores valores en la mayoría de los atributos morfológicos, aunque los efectos fueron estadísticamente similares a los de T3, T5 y T6.

Palabras clave: crecimiento, hidrolizado de proteina, nutrición vegetal, procesamiento de imágenes, Solanum lycopersicum L.

\section{SUMMARY}

In the context of sustainable agriculture, the use of organic fertilizers is a viable option given the environmental impact caused by conventional fertilizers. The objective of this work was to evaluate the effect of organic fertilizers as a source of $\mathrm{N}$ on growth, yield, quality, and morphological traits in grape tomato fruits. Seven fertilization treatments were

Cita recomendada:

González-Fuentes, J. A., Lozano-Cavazos, C. J., Preciado-Rangel, P., Troyo-Diéguez, E., Rojas-Duarte, A. y Rodríguez-Ortiz, J. C. (2021). Fertilización orgánica contra convencional en el rendimiento, atributos morfológicos y calidad de fruto de tomate uva en un sistema de subirrigación no recirculante. Terra Latinoamericana, 39, 1-16. e897. https://doi.org/10.28940/terra.v39i0.897 
evaluated, which consisted of an inorganic nutrient solution (Steiner solution) and different mixtures of various OMRI certified nitrogen fertilizers of animal origin at a concentration ranging 100 to $120 \%$. The results showed that $\mathrm{T} 5$ and $\mathrm{T} 6$ organic treatments showed a growth similar to the conventional treatment (T7). The SPAD index was $13.8 \%$ higher in organic treatments compared to $\mathrm{T} 7$ inorganic treatment. In the fruit production parameters, the $\mathrm{T} 5$ organic treatment was similar to the conventional treatment in the number of fruits per cluster, although in fresh fruit weight, yield, and longitudinal diameter, the conventional treatment surpassed the organic treatments by 21 , 31.6, and 5.8\%; whereas, the transverse diameter was similar in treatments $\mathrm{T} 1$ and $\mathrm{T} 7$. The fruit firmness was higher in T1 and T4 organic treatments, surpassing the inorganic treatment by 10.3 and $6 \%$, respectively. Total soluble solids were higher in T4 organic treatment, surpassing the $\mathrm{T} 7$ inorganic treatment by $18 \%$. The T7 inorganic treatment had higher values in most of the morphological attributes, although the effects were statistically similar to those of T3, T5, and T6.

Index words: growth, protein hydrolysate, plant nutrition, image processing, Solanum lycopersicum L.

\section{INTRODUCCIÓN}

La producción convencional que involucra el uso excesivo de fertilizantes sintéticos y plaguicidas, es esencial para garantizar la seguridad alimentaria de la creciente población humana (Huang, Pray, y Rozelle, 2002). Sin embargo, dicho método de producción ha ocasionado importantes daños directos e indirectos al medio ambiente (Beman, Arrigo y Matson, 2005). Por lo anterior, es necesario adoptar estrategias que proporcionen una nutrición óptima a las plantas con una menor contaminación ambiental, pero que a la vez permitan mejorar los rendimientos de cultivo (Haley y Reed, 2004). La agricultura orgánica es un sistema de producción que sustituye el uso de fertilizantes sintéticos por insumos orgánicos debidamente inspeccionados y certificados para proporcionar los nutrimentos necesarios a los cultivos (Muller et al., 2017). Este sistema tiene como principales desafíos aumentar los rendimientos de los cultivos, reducir la contaminación ambiental y mejorar el manejo de nutrimentos, promoviendo la equidad social en el campo (Tuomisto,
Hodge, Riordan y Macdonald, 2012). Sin embargo, los fertilizantes orgánicos dependen de la degradación microbiana y química para convertir los nutrimentos unidos orgánicamente en iones disponibles para las plantas, lo anterior origina problemas para hacer coincidir la disponibilidad de nutrimentos disueltos en el medio de cultivo con la demanda de las plantas, (Cavigelli, Teasdale y Conklin, 2008). Además, se ha indicado que debido a que los fertilizantes orgánicos comerciales certificados derivan de diferentes fuentes (vegetales, animales o minerales), estos pueden producir un efecto diferente en los cultivos (Khandaker, Rohani, Dalorima y Mat, 2017). Por otro lado, una innovación prometedora y respetuosa con el medio ambiente sería el uso de bioestimulantes naturales de plantas (BP) que mejoran la floración, el crecimiento de las plantas, el cuajado, la productividad de los cultivos y la eficiencia en el uso de nutrientes (EUN), varios de ellos presentes en las enmiendas orgánicas, incluyendo los ácidos húmicos y otros (Colla y Rouphael, 2015). Debido a la relevancia de este tema, aunado a la escasa información sobre la fertilización orgánica en tomates de especialidad, el presente estudio tuvo como objetivo determinar el efecto de diferentes fuentes orgánicas de $\mathrm{N}$ y una proteína hidrolizada a base de pescado sobre el crecimiento, rendimiento, atributos morfológicos y de calidad de fruto en tomate uva cultivado en condiciones de invernadero bajo el sistema de subirrigación no recirculante.

\section{MATERIALES Y MÉTODOS}

\section{Germoplasma y Condiciones Experimentales}

El estudio se realizó en 2015 en un invernadero en la Universidad Autónoma Agraria Antonio Narro, en Saltillo, Coahuila, México $\left(25^{\circ} 21^{\prime} \mathrm{N}, 101^{\circ} 02^{\prime} \mathrm{O}\right.$, a una altitud de $1759 \mathrm{~m}$ ). Las condiciones ambientales durante el experimento incluyeron una temperatura promedio diaria de $21.88{ }^{\circ} \mathrm{C}$ y humedad relativa promedio de $60.40 \%$. Se emplearon plántulas de tomate uva (Solanum lycopersicum L.) cv. Luciplus con cuatro hojas bien desarrolladas, las cuales fueron trasplantadas el 17 de julio de 2015 en contenedores de polietileno negro de $15 \mathrm{~L}$, que fueron llenados previamente con un sustrato a base de mezcla de sphagnum peat y perlita (80:20, v:v). Cada planta se cultivó a dos tallos. Se emplearon siete tratamientos de 
fertilización: seis tratamientos de fertilización orgánica y uno de fertilización inorgánica (control). Para la fertilización inorgánica se empleó la solución nutritiva propuesta por Steiner (1984) al 100\% (meq $\left.\cdot \mathrm{L}^{-1}\right): 12 \mathrm{NO}_{3}$, $1 \mathrm{H}_{2} \mathrm{PO}_{4}, 7 \mathrm{~K}, 4 \mathrm{Mg}, 9$ Ca y $7 \mathrm{SO}_{4}$ Para los tratamientos de fertilización orgánica se emplearon fertilizantes orgánicos aprobados por OMRI (Organic Materials Review Institute), variando la dosis (100 y 120\%) y la fuente de nitrógeno $\left(\mathrm{FON}^{\circledR} \mathrm{HCP}\right.$, VIGILANTE ${ }^{\circledR}$, PHYTAFISH $^{\circledR}$ ), mientras que las fuentes empleadas para suplementar el resto de los nutrimentos $(\mathrm{P}, \mathrm{K}, \mathrm{Ca}$, $\mathrm{Mg}$ y S) fueron los mismos en todos los tratamientos orgánicos (Cuadro 1). La cantidad aplicada de material orgánico se calculó para cumplir con los niveles equivalentes de nitrógeno $(\mathrm{N})$, fósforo $(\mathrm{P})$, potasio $(\mathrm{K})$, calcio $(\mathrm{Ca})$, magnesio $(\mathrm{Mg})$ y azufre $(\mathrm{S})$ aplicados con la solución nutritiva propuesta por Steiner al 100 y $120 \%$. La composición de nutrimentos aplicados por tratamiento orgánico se detalla en el Cuadro 2; las soluciones nutritivas se aplicaron a los tres días después del trasplante. La conductividad eléctrica (CE) de las soluciones nutritivas varió de $2.1 \mathrm{a} 2.3 \mathrm{mS} \mathrm{cm}^{-1} \mathrm{y}$ el pH de 5.9 a 6.2 en todos los tratamientos evaluados. Se empleó el método de riego de subirrigación no recirculante, colocando cada unidad experimental dentro de una bandeja de plástico rígido $(40 \mathrm{~cm}$ de diámetro y $15 \mathrm{de} \mathrm{cm}$ de altura). Para la aplicación manual del riego, se añadió a cada bandeja de 2 a $6 \mathrm{~L}$ de solución nutritiva, dependiendo de la etapa fenológica del cultivo, para que el agua se moviera por capilaridad a la parte superior del sustrato. Se utilizó el producto pH ORGÁNIC (Altiara ${ }^{\circledR}$, listado en OMRI) para ajustar el $\mathrm{pH}$ de las soluciones orgánicas a $5.8 \pm 0.1$ antes de cada riego; por su parte, para la fertilización inorgánica se empleó $\mathrm{H}_{2} \mathrm{SO}_{4}$. En el manejo preventivo para el control de plagas y enfermedades se utilizaron

Cuadro 1. Tratamientos de fertilización aplicados al tomate uva bajo el sistema de subirrigación no recirculante.

Table 1. Fertilization treatments applied to grape tomato under non-recirculating subirrigation.

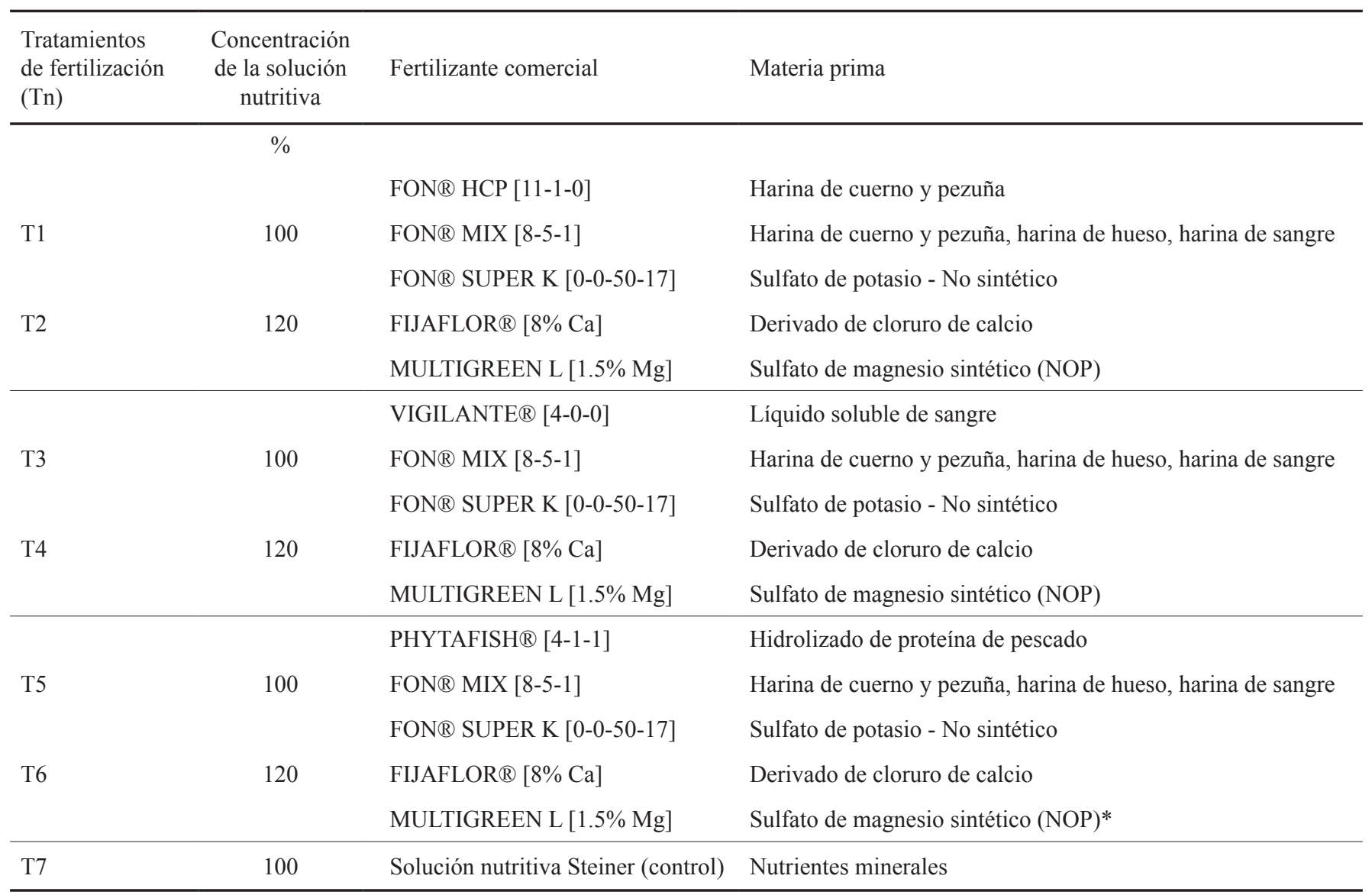

* USDA - Programa Orgánico Nacional, para su uso en cualquier sistema de producción orgánica certificada según las normas NOP.

* USDA - National Organic Program, for use in any organic production system certified according to NOP standards. 
bioplaguicidas orgánicos de origen botánico y microbiano de GreenCorp Biorganiks de México ${ }^{\circledR}$, aprobados por IFOAM y OMRI.

\section{Parámetros de Crecimiento y SPAD}

Para determinar estas variables, se tomaron seis plantas por tratamiento. Estos parámetros se evaluaron cada 30 días después del trasplante (DDT). La altura de la planta se midió desde la superficie del sustrato hasta el ápice de ambos tallos. El diámetro del tallo se midió a $3 \mathrm{~cm}$ del origen de cada tallo utilizando un calibrador digital (Mitutoyo ${ }^{\circledR}$ ). Los niveles relativos de clorofila (valores SPAD) se determinaron en la última hoja completamente expandida utilizando un medidor portátil SPAD (Modelo SPAD-502, Minolta Co., Ltd.). Las mediciones se realizaron entre las 7:00 y las 9:00 am.

\section{Parámetros de Rendimiento}

El número de frutos por racimo, peso de fruto $\mathrm{y}$ rendimiento de fruto por racimo se determinó mediante el conteo y peso de los frutos por racimo producidos en ambos tallos en seis plantas por tratamiento, considerando los frutos del $1^{\circ}$ al $4^{\circ}$ racimo.

\section{Parámetros de Calidad de Fruto}

Los tomates se cosecharon en etapa de color rojo claro ( $>90 \%$ de rojo con epicarpio opaco), para lo cual se seleccionaron aleatoriamente cinco frutos representativos por racimo en ambos tallos de 6 plantas seleccionadas por tratamiento. El diámetro longitudinal y el transversal de fruto se midieron utilizando un vernier digital electrónico (Mitutoyo ${ }^{\circledR}$ ). La firmeza de fruto se determinó con un medidor de dureza de fruta

Cuadro 2. Composición de soluciones de nutrientes orgánicos aplicadas a tomates uva bajo el sistema de subirrigación no recirculante. Table 2. Composition of organic nutrient solutions applied to grape tomatoes under non-recirculating subirrigation.

\begin{tabular}{|c|c|c|c|c|c|c|c|c|c|c|c|c|}
\hline \multirow{3}{*}{ Fertilizante comercial } & \multicolumn{12}{|c|}{ Nutrientes minerales aplicados en cada tiempo de riego $\left(\mathrm{g} \mathrm{L}^{-1} / \mathrm{ml} \mathrm{L}^{-1}\right)$} \\
\hline & \multicolumn{2}{|c|}{$\mathrm{N}$} & \multicolumn{2}{|c|}{$\mathrm{P}$} & \multicolumn{2}{|c|}{$\mathrm{K}$} & \multicolumn{2}{|c|}{$\mathrm{S}$} & \multicolumn{2}{|c|}{$\mathrm{Ca}$} & \multicolumn{2}{|c|}{$\mathrm{Mg}$} \\
\hline & 100 & 120 & 100 & 120 & 100 & 120 & 100 & 120 & 100 & 120 & 100 & 120 \\
\hline & $-\cdots$ & $-\cdots$ & $-\cdots$ & $\cdots$ & $-\cdots$ & -- & -- & $-\cdots$ & -- & $-\cdots$ & $-\cdots$ & $-\cdots$ \\
\hline $\mathrm{FON}^{\circledR} \mathrm{HCP}[11-1-0]$ & 0.08 & 0.09 & 0 & 0 & - & - & - & - & - & - & - & - \\
\hline $\mathrm{FON}^{\circledR} \mathrm{MIX}[8-5-1]$ & 0.1 & 0.13 & 0.03 & 0.03 & 0.01 & 0.01 & - & - & - & - & - & - \\
\hline $\mathrm{FON}^{\circledR}$ SUPER K [0-0-50-17] & - & - & - & - & 0.26 & 0.32 & 0.11 & 0.13 & - & - & - & - \\
\hline FIJAFLOR $^{\circledR}[8 \% \mathrm{Ca}]$ & - & - & - & - & - & - & - & - & 1.2 & 1.44 & - & - \\
\hline MULTIGREEN L [1.5\% Mg] & - & - & - & - & - & - & - & - & - & - & 0.98 & 1.18 \\
\hline VIGILANTE $^{\circledR}[4-0-0]$ & 0.05 & 0.06 & - & - & - & - & - & - & - & - & - & - \\
\hline $\mathrm{FON}^{\circledR} \mathrm{MIX}[8-5-1]$ & 0.12 & 0.14 & 0.03 & 0.04 & 0.01 & 0.01 & - & - & - & - & - & - \\
\hline $\mathrm{FON}^{\circledR}$ SUPER K [0-0-50-17] & - & - & - & - & 0.26 & 0.31 & 0.11 & 0.13 & - & - & - & - \\
\hline FIJAFLOR $^{\circledR}[8 \% \mathrm{Ca}]$ & - & - & - & - & - & - & - & - & 1.2 & 1.44 & - & - \\
\hline MULTIGREEN L [1.5\% Mg] & - & - & - & - & - & - & - & - & - & - & 0.98 & 1.18 \\
\hline PHYTAFISH $^{\circledR}$ [4-1-1] & 0.09 & 0.11 & 0.01 & 0.01 & 0.02 & 0.02 & - & - & - & - & - & - \\
\hline $\mathrm{FON}^{\circledR} \operatorname{MIX}[8-5-1]$ & 0.08 & 0.09 & 0.02 & 0.03 & 0.01 & 0.01 & - & - & - & - & - & - \\
\hline $\mathrm{FON}^{\circledR}$ SUPER K [0-0-50-17] & - & - & - & - & 0.25 & 0.3 & 0.1 & 0.12 & - & - & - & - \\
\hline FIJAFLOR $^{\circledR}[8 \% \mathrm{Ca}]$ & - & - & - & - & - & - & - & - & 1.2 & 1.44 & - & - \\
\hline MULTIGREEN L [1.5\% Mg] & - & - & - & - & - & - & - & - & - & - & 0.98 & 1.18 \\
\hline
\end{tabular}


(Lutron Electronic Enterprise Co., Ltd, Modelo FR5120). La determinación de sólidos solubles totales se realizó colocando una gota de jugo de fruta en el prisma de un refractómetro digital (ATAGO ${ }^{\circledR}$, PAL-1) con compensación automática de temperatura.

\section{Medición de Lixiviado}

Los lixiviados se evaluaron en tres plantas por tratamiento; la primera evaluación se realizó por primera vez a los cinco días después del trasplante (DDT), cuando se inició el riego con las soluciones nutritivas. Las evaluaciones posteriores se realizaron cada mes después del trasplante hasta el final de la temporada de crecimiento, tomando la lectura dos horas después de haber realizado cada riego con las soluciones nutritivas correspondientes a cada tratamiento. Se determinó el $\mathrm{pH}, \mathrm{CE}, \mathrm{NO}_{3}^{-} \mathrm{y} \mathrm{K}^{+}$con ayuda de ionómetros portátiles (Horiba LAQUA twin).

\section{Caracterización de la Forma del Fruto}

Se digitalizó un protocolo para la digitalización de la fruta de tomate uva y el posterior análisis semiautomático de atributos morfológicos con el paquete de software Tomato Analyzer (TA) versión 4.0 (Rodríguez et al., 2010). En cada tratamiento evaluado, se cosecharon cinco frutas de cada racimo en la etapa de maduración, con frutos completamente rojos, los cuales fueron llevados al laboratorio y sometidos a lavado. Posteriormente, los frutos se cortaron longitudinalmente a través del centro y fueron colocados en una HP Scanjet G3110 (HewlettPackard, Palo Alto, CA, EE. UU.) a una resolución de 300 dpi, la cual se cubrió con una caja para minimizar el efecto de sombra y proporcionar un fondo negro, y posteriormente fueron sometidos al análisis fenotípico. Diez rasgos de forma de fruta fueron clasificados en tres categorías del software: medición básica (6), índice de forma de fruto (2) y sección latitudinal (2) (Cuadro 3).

\section{Diseño Experimental y Análisis Estadístico}

El diseño experimental fue bloques al azar, con tres repeticiones por tratamiento y dos plantas por repetición. Los datos se sometieron a análisis de varianza y la comparación de medias se realizó mediante la prueba DMS $(\alpha \leq 0.05)$ con el programa de estadística SAS (Statistical Analysis Systems) versión 9.4.

Cuadro 3. Descriptores morfológicos de fruto de tomate uva.

Table 3. Grape tomato fruit shape descriptors.

\begin{tabular}{|c|c|}
\hline Descriptores morfológicos & Unidades / descripción \\
\hline \multicolumn{2}{|r|}{ Medidas básicas } \\
\hline Perímetro & Longitud del perímetro $(\mathrm{cm})$ \\
\hline Área & Área del fruto $\left(\mathrm{cm}^{2}\right)$ \\
\hline Ancho a media altura & Anchura medida a la mitad de la altura de la fruta $(\mathrm{cm})$ \\
\hline Ancho máximo & Distancia horizontal máxima de la fruta $(\mathrm{cm})$ \\
\hline Altura a media anchura & Altura medida a la mitad de la anchura de la fruta $(\mathrm{cm})$ \\
\hline Altura máxima & La distancia vertical máxima de la fruta $(\mathrm{cm})$ \\
\hline \multicolumn{2}{|r|}{ Índice de forma de fruto } \\
\hline Índice de forma de fruto (I) & Relación entre la altura máxima y la anchura máxima \\
\hline Índice de forma de fruto (II) & Relación entre la anchura a media altura $(\mathrm{cm})$ y altura a media anchura $(\mathrm{cm})$ \\
\hline \multicolumn{2}{|r|}{ Sección latitudinal } \\
\hline Área de pericarpio & Área entre el límite del pericarpio y el perímetro del fruto $\left(\mathrm{cm}^{2}\right)$ \\
\hline Grosor de pericarpio & Área del pericarpio dividido por el promedio de la longitud del límite del pericarpio y el perímetro del fruto \\
\hline
\end{tabular}

Los detalles para la medición de dichos atributos con el citado analizador fueron descritos por Gonzalo y Van der Knaap (2008) y Rodríguez et al. (2010).

The details for measuring these attributes with the aforementioned analyzer were described by Gonzalo and Van der Knaap (2008) and Rodríguez et al. (2010). 


\section{RESULTADOS Y DISCUSIÓN}

La fertilización inorgánica y orgánica, según la fuente de $\mathrm{N}$ y dosis, causaron diferentes respuestas en la altura de planta, diámetro de tallo e índice SPAD durante el crecimiento de las plantas de tomate uva.
La altura de planta presentó diferencias estadísticas en los meses evaluados (Cuadro 4), observándose que un mes después del trasplante los efectos de los tratamientos orgánicos T4, T5 y T6 fueron similares al tratamiento inorgánico (T7), reflejando éste, mayor altura de planta que el resto de los tratamientos.

Cuadro 4. Efecto de la fertilización orgánica e inorgánica en altura de planta, diámetro de tallo y fluorescencia relativa de clorofila (índice SPAD) en tomate uva bajo subirrigación no recirculante.

Table 4. Effect of organic and inorganic fertilization on plant height, stem diameter and relative chlorophyll fluorescence (SPAD index) on grape tomato under non-recirculating subirrigation.

\begin{tabular}{|c|c|c|c|c|c|}
\hline \multirow{2}{*}{ Tratamientos } & \multicolumn{5}{|c|}{ Mes de evaluación } \\
\hline & 1 & 2 & 3 & 4 & 5 \\
\hline & \multicolumn{5}{|c|}{ Altura de planta $(\mathrm{cm})$} \\
\hline $\mathrm{T} 1$ & $24.13 c$ & $86.90 \mathrm{c}$ & $144.63 b$ & $180.90 \mathrm{bc}$ & $198.98 b c$ \\
\hline $\mathrm{T} 2$ & $33.38 \mathrm{c}$ & $97.08 \mathrm{bc}$ & $146.53 b$ & $188.23 \mathrm{bc}$ & $207.05 b c$ \\
\hline $\mathrm{T} 3$ & $35.18 \mathrm{bc}$ & $88.13 \mathrm{c}$ & $139.10 \mathrm{~b}$ & $182.70 \mathrm{bc}$ & $195.13 b c$ \\
\hline $\mathrm{T} 4$ & $35.55 \mathrm{abc}$ & $95.55 b c$ & $130.83 b$ & $146.83 c$ & $159.50 \mathrm{c}$ \\
\hline T5 & $37.50 \mathrm{abc}$ & $110.40 \mathrm{ab}$ & $159.38 \mathrm{ab}$ & $200.55 \mathrm{ab}$ & $218.63 \mathrm{ab}$ \\
\hline T6 & $49.05 \mathrm{ab}$ & $95.70 \mathrm{bc}$ & $161.68 \mathrm{ab}$ & $198.83 \mathrm{ab}$ & $225.53 \mathrm{ab}$ \\
\hline $\mathrm{T} 7$ & $51.40 \mathrm{a}$ & $122.93 a$ & $188.23 \mathrm{a}$ & $239.30 \mathrm{a}$ & $266.90 \mathrm{a}$ \\
\hline \multirow[t]{2}{*}{ ANOVA $\mathrm{P} \leq$} & 0.018 & 0.031 & 0.025 & 0.027 & 0.018 \\
\hline & \multicolumn{5}{|c|}{ Diámetro de tallo $(\mathrm{cm})$} \\
\hline $\mathrm{T} 1$ & $0.49 \mathrm{a}$ & $0.93 \mathrm{a}$ & $0.95 \mathrm{ab}$ & $0.99 \mathrm{ab}$ & $1.03 \mathrm{abc}$ \\
\hline $\mathrm{T} 2$ & $0.53 \mathrm{a}$ & $0.76 \mathrm{bc}$ & $0.95 \mathrm{ab}$ & $0.99 \mathrm{ab}$ & $0.98 \mathrm{bc}$ \\
\hline $\mathrm{T} 3$ & $0.55 \mathrm{a}$ & $0.72 \mathrm{~cd}$ & $0.89 b c$ & $0.99 \mathrm{ab}$ & $0.99 \mathrm{bc}$ \\
\hline $\mathrm{T} 4$ & $0.52 \mathrm{a}$ & $0.67 \mathrm{~d}$ & $0.74 d$ & $0.93 b c$ & $0.91 \mathrm{c}$ \\
\hline T5 & $0.61 \mathrm{a}$ & $0.8 \mathrm{ab}$ & $0.99 \mathrm{ab}$ & $1.04 \mathrm{ab}$ & $1.09 \mathrm{ab}$ \\
\hline T6 & $0.46 \mathrm{a}$ & $0.81 \mathrm{bc}$ & $0.79 \mathrm{~cd}$ & $0.84 \mathrm{c}$ & $0.90 \mathrm{c}$ \\
\hline $\mathrm{T} 7$ & $0.62 \mathrm{a}$ & $0.96 \mathrm{a}$ & $1.07 \mathrm{a}$ & $1.12 \mathrm{a}$ & $1.17 \mathrm{a}$ \\
\hline \multirow[t]{2}{*}{ ANOVA $\mathrm{P} \leq$} & 0.542 & 0.001 & 0.001 & 0.033 & 0.026 \\
\hline & \multicolumn{5}{|c|}{ SPAD } \\
\hline $\mathrm{T} 1$ & $39.77 \mathrm{a}$ & $45.13 \mathrm{a}$ & $48.3 \mathrm{a}$ & $46.88 \mathrm{a}$ & $49.50 \mathrm{a}$ \\
\hline $\mathrm{T} 2$ & $39.00 \mathrm{a}$ & $46.23 \mathrm{a}$ & $51.33 \mathrm{a}$ & $45.58 \mathrm{ab}$ & $45.98 \mathrm{ab}$ \\
\hline $\mathrm{T} 3$ & $40.38 \mathrm{a}$ & $46.93 a$ & $52.33 \mathrm{a}$ & $47.50 \mathrm{a}$ & $44.20 \mathrm{~b}$ \\
\hline $\mathrm{T} 4$ & $41.30 \mathrm{a}$ & $50.18 \mathrm{a}$ & $49.85 \mathrm{a}$ & $46.18 \mathrm{ab}$ & $43.98 \mathrm{~b}$ \\
\hline T5 & $35.75 \mathrm{a}$ & $47.38 \mathrm{a}$ & $49.40 \mathrm{a}$ & $44.20 \mathrm{~b}$ & $47.93 \mathrm{a}$ \\
\hline T6 & $40.38 \mathrm{a}$ & $45.32 \mathrm{a}$ & $48.58 \mathrm{a}$ & $46.90 \mathrm{a}$ & $43.55 b c$ \\
\hline $\mathrm{T} 7$ & $38.65 \mathrm{a}$ & $49.33 \mathrm{a}$ & $52.18 \mathrm{a}$ & $41.23 \mathrm{c}$ & $39.90 \mathrm{c}$ \\
\hline ANOVA $\mathrm{P} \leq$ & 0.928 & 0.56 & 0.484 & 0.001 & 0.001 \\
\hline
\end{tabular}

Medias en la misma columna con la misma letra no presentan diferencias significativas, según la prueba DMS $(P \leq 0.05)$. ANOVA $=$ análisis de varianza. Means in the same column with the same letter do not show significant differences, according to the LSD test $(P \leq 0.05)$. ANOVA $=$ analysis of variance. 
En el segundo mes los tratamientos T5 y $\mathrm{T} 7$ presentaron mayor valor; pero, del tercer al quinto mes después del trasplante los efectos de los tratamientos orgánicos T5 y T6 fueron similares a T7, con una altura de planta al final del experimento de 218.63, 225.53 y $266.90 \mathrm{~cm}$, respectivamente. El diámetro de tallo presentó diferencia significativa del segundo al quinto mes después del trasplante; los tratamientos orgánicos T1 y T5 presentaron un diámetro de tallo similar a T7 durante dichos meses, con valores al final del experimento de $1.03,1.09$ y $1.17 \mathrm{~cm}$, respectivamente. El índice SPAD presentó diferencias significativas únicamente en el cuarto y quinto mes después del trasplante, donde los tratamientos orgánicos superaron al tratamiento $\mathrm{T} 7$ en el cuarto mes; asimismo, en el quinto mes de evaluación, a excepción del T6, los tratamientos orgánicos superaron al tratamiento inorgánico T7 (Cuadro 4).

La aplicación de fertilización orgánica modificó la altura de planta de tomate uva al final de la temporada de crecimiento del cultivo; en promedio, los tratamientos orgánicos registraron $200.8 \mathrm{~cm}$ de longitud y $0.98 \mathrm{~cm}$ de diámetro de tallo, mientras que el inorgánico superó a los tratamientos orgánicos tanto en longitud como en diámetro de tallo en un 24.77 y $16.24 \%$, respectivamente. Aun cuando varió únicamente una fuente de $\mathrm{N}$ en los tratamientos orgánicos, se observaron plantas de mayor altura en las etapas evaluadas en los tratamientos T5 y T6. Dichos tratamientos variaron del resto de los tratamientos orgánicos por la fuente de $\mathrm{N}$ a base de hidrolizados de proteína de pescado con soluciones nutritivas al 100 y $120 \%$, respectivamente. A su vez, las plantas con mayor diámetro de tallo desde el segundo al quinto mes de evaluación se presentaron en los tratamientos $\mathrm{T} 1$ y $\mathrm{T} 5$, siendo la fuente de nitrógeno a base de harina de cuerno y pezuña e hidrolizado de proteína de pescado, respectivamente, ambas a una concentración al 100\%. La mejor respuesta del tratamiento T5 pudo responder al hidrolizado de proteína de pescado, ya que se ha reportado que mejora el crecimiento de las plantas (Colla et al., 2014; Xu y Mou, 2017), atribuyéndolo al aumento de la absorción, asimilación y metabolismo de los nutrientes como resultado del aumento de la actividad microbiana del suelo, la mejora de la movilidad y la solubilidad de los micronutrientes ( $\mathrm{Fe}, \mathrm{Mn}$ y $\mathrm{Zn}$ ) (Cristiano et al., 2018; Ertani, Schiavon, Muscolo y Nardi, 2013; Lucini et al., 2015; Santi, Zamboni, Varanini y Pandolfini, 2017). Al finalizar el ciclo experimental, los tratamientos orgánicos presentaron mayores valores SPAD, superando al inorgánico en 13.8\%. Bergstrand, Löfkvist y Asp (2018) indicaron que la menor concentración de clorofila en plantas fertilizadas de manera orgánica pudo deberse a la lenta liberación de nitrógeno. En este trabajo se observaron diferencias significativas en el contenido relativo de clorofila hasta el cuarto mes de evaluación, lo que pudo deberse al insuficiente suministro o disponibilidad de $\mathrm{N}$ antes de dicho período en el caso de los tratamientos orgánicos, ya que su disponibilidad no es inmediata debido a que se requiere de un proceso de mineralización. En este contexto, la cantidad y la tasa de mineralización de $\mathrm{N}$ en fertilizantes orgánicos está en función de las condiciones ambientales, así como de la composición del suelo o sustrato (Agehara y Warncke, 2005; Bi, Evans, Spiers y Witcher, 2010; Stadler et al., 2006). Asimismo, Gaskell y Smith (2007), señalaron que los sub-productos de origen animal son ricos en proteínas $\mathrm{y}$, por lo tanto, ofrecen una buena fuente de $\mathrm{N}$ para los cultivos. Esto último, puede explicar el hecho de que los tratamientos orgánicos tuvieron un efecto similar en cuanto a valores SPAD con respecto al tratamiento inorgánico, principalmente al inicio del experimento.

El número de frutos por racimo, a excepción del segundo racimo, y el número de frutos total por planta fueron influenciados de manera significativa por los tratamientos (Cuadro 5), con mayor número de frutos por planta en los tratamientos T5 (104) y T7 (111). Sin embargo, en el primer racimo evaluado el tratamiento inorgánico T7 presentó el mayor número de frutos por racimo que los tratamientos orgánicos, en tanto que en el tercer racimo los tratamientos orgánicos $\mathrm{T} 4 \mathrm{y}$ T5 presentaron similar número de frutos por racimo al tratamiento inorgánico $\mathrm{T} 7$, superando al resto de los tratamientos. A su vez, en el cuarto racimo los tratamientos orgánicos T3, T4 y T5 presentaron similar número de frutos por racimo al inorgánico $\mathrm{T} 7$. El peso fresco de fruto presentó diferencia significativa en cada uno de los racimos evaluados, así como en el promedio de éste parámetro, presentando en el primer, segundo y tercer racimo evaluado un mayor peso fresco por fruto en el tratamiento inorgánico T7; sin embargo, en el cuarto racimo los tratamientos orgánicos T1, T5 y T6 presentaron un peso fresco por fruto similar al tratamiento inorgánico $\mathrm{T} 7$; mientras que, en promedio, el tratamiento inorgánico T7 superó por un $21 \%$ el peso fresco por fruto obtenido en los tratamientos orgánicos (Cuadro 5). El rendimiento de los racimos evaluados y el rendimiento total por planta fueron 
afectados significativamente por los tratamientos, obteniéndose un rendimiento por planta mayor en el tratamiento $\mathrm{T} 7$, superando a los tratamientos orgánicos en $31.6 \%$. Sin embargo, durante la evaluación de cada uno de los racimos se observó que en el primer racimo el rendimiento fue superior en el tratamiento $\mathrm{T} 7$; mientras que, algunos de los tratamientos orgánicos en el segundo (T1 y T5), tercer (T1, T2 y T5) y cuarto (T2, $\mathrm{T} 3, \mathrm{~T} 4$ y T5) racimo presentaron un rendimiento por racimo similar al tratamiento inorgánico T7 (Cuadro 5).

Cuadro 5. Efecto de la fertilización orgánica e inorgánica en el número de frutos por racimo, peso fresco por fruto y rendimiento total por racimo en tomate uva bajo subirrigación no recirculante.

Table 5. Effect of organic and inorganic fertilization on fruit number per cluster, fresh weight per fruit and total yield per cluster on grape tomato under non-recirculating subirrigation.

\begin{tabular}{|c|c|c|c|c|c|}
\hline \multirow{2}{*}{ Tratamientos } & \multicolumn{4}{|c|}{ Racimos } & \multirow{2}{*}{ Total } \\
\hline & 1 & 2 & 3 & 4 & \\
\hline \multicolumn{6}{|c|}{ Número de frutos por racimo } \\
\hline $\mathrm{T} 1$ & $22.50 \mathrm{~b}$ & $28.50 \mathrm{a}$ & $21.33 b$ & $16.50 \mathrm{c}$ & $88.83 \mathrm{~cd}$ \\
\hline $\mathrm{T} 2$ & $20.25 b$ & $21.00 \mathrm{a}$ & $20.00 \mathrm{~b}$ & $18.50 \mathrm{bc}$ & $79.75 \mathrm{~cd}$ \\
\hline $\mathrm{T} 3$ & $22.25 b$ & $25.75 \mathrm{a}$ & $18.67 \mathrm{~b}$ & $22.68 \mathrm{a}$ & $89.33 \mathrm{~cd}$ \\
\hline $\mathrm{T} 4$ & $19.00 \mathrm{~b}$ & $23.50 \mathrm{a}$ & $28.33 \mathrm{a}$ & $21.00 \mathrm{ab}$ & $91.83 \mathrm{bc}$ \\
\hline T5 & $23.50 \mathrm{~b}$ & $29.50 \mathrm{a}$ & $30.33 a$ & $21.00 \mathrm{ab}$ & $104.44 \mathrm{ab}$ \\
\hline T6 & $20.75 b$ & $20.00 \mathrm{a}$ & $20.00 \mathrm{~b}$ & $16.00 \mathrm{c}$ & $76.75 d$ \\
\hline $\mathrm{T} 7$ & $34.25 \mathrm{a}$ & $26.75 \mathrm{a}$ & $28.50 \mathrm{a}$ & $21.68 \mathrm{ab}$ & $111.17 \mathrm{a}$ \\
\hline ANOVA $P \leq$ & 0.024 & 0.346 & 0.002 & 0.005 & 0.001 \\
\hline \multicolumn{5}{|c|}{ Peso fresco por fruto $(\mathrm{g})$} & Promedio \\
\hline $\mathrm{T} 1$ & $9.44 b$ & $8.64 \mathrm{~b}$ & $7.69 \mathrm{~b}$ & $8.17 \mathrm{ab}$ & $8.48 b$ \\
\hline $\mathrm{T} 2$ & $9.68 b$ & $8.90 \mathrm{~b}$ & $7.39 b$ & $7.90 \mathrm{bc}$ & $8.47 \mathrm{~b}$ \\
\hline $\mathrm{T} 3$ & $9.06 \mathrm{~b}$ & $8.07 \mathrm{~b}$ & $7.73 b$ & $7.40 \mathrm{~d}$ & $8.07 \mathrm{~b}$ \\
\hline $\mathrm{T} 4$ & $7.34 \mathrm{c}$ & $5.75 \mathrm{c}$ & $6.29 c$ & $7.53 \mathrm{~cd}$ & $6.73 c$ \\
\hline T5 & $9.76 \mathrm{~b}$ & $9.01 \mathrm{~b}$ & $6.89 \mathrm{bc}$ & $8.40 \mathrm{a}$ & $8.52 b$ \\
\hline T6 & $9.25 b$ & $8.97 \mathrm{~b}$ & $7.48 \mathrm{~b}$ & $8.48 \mathrm{a}$ & $8.55 b$ \\
\hline $\mathrm{T} 7$ & $11.85 \mathrm{a}$ & $11.48 \mathrm{a}$ & $9.71 \mathrm{a}$ & $8.19 \mathrm{ab}$ & $10.31 \mathrm{a}$ \\
\hline ANOVA $P \leq$ & 0.001 & 0.001 & 0.001 & 0.001 & 0.001 \\
\hline \multicolumn{5}{|c|}{ Rendimiento por racimo $(\mathrm{g})$} & Total \\
\hline $\mathrm{T} 1$ & $211.70 \mathrm{~b}$ & $239.58 \mathrm{abc}$ & $229.03 \mathrm{a}$ & $129.68 b$ & $809.98 b c$ \\
\hline $\mathrm{T} 2$ & $215.38 b$ & $190.78 \mathrm{bcd}$ & $195.70 \mathrm{abc}$ & $150.83 \mathrm{ab}$ & $752.68 \mathrm{bcd}$ \\
\hline $\mathrm{T} 3$ & $190.03 \mathrm{bc}$ & $217.08 \mathrm{bc}$ & $145.23 \mathrm{~cd}$ & $151.53 \mathrm{ab}$ & $703.87 \mathrm{~cd}$ \\
\hline $\mathrm{T} 4$ & $138.48 \mathrm{c}$ & $134.68 d$ & $129.60 d$ & $163.88 \mathrm{a}$ & $565.62 \mathrm{e}$ \\
\hline T5 & $195.60 \mathrm{bc}$ & $258.88 \mathrm{ab}$ & $238.43 a$ & $164.30 \mathrm{a}$ & $857.20 b$ \\
\hline $\mathrm{T} 6$ & $188.93 \mathrm{bc}$ & $183.40 \mathrm{~cd}$ & $164.13 \mathrm{bcd}$ & $134.60 \mathrm{~b}$ & $670.06 \mathrm{de}$ \\
\hline $\mathrm{T} 7$ & $374.09 \mathrm{a}$ & $302.85 \mathrm{a}$ & $211.17 \mathrm{ab}$ & $174.33 \mathrm{a}$ & $1062.43 a$ \\
\hline ANOVA $P \leq$ & 0.001 & 0.003 & 0.008 & 0.023 & 0.001 \\
\hline
\end{tabular}

Medias en la misma columna con la misma letra no presentan diferencias significativas, según la prueba DMS $(P \leq 0.05)$. ANOVA= análisis de varianza. Means in the same column with the same letter do not show significant differences, according to the LSD test $(P \leq 0.05)$. ANOVA $=$ analysis of variance. 
Una de las limitantes en la producción orgánica de cultivos es precisamente los bajos rendimientos comparado a los sistemas de fertilización inorgánica, en el que se ha reportado un rendimiento alrededor de $20 \%$ menor que el obtenido con fertilizantes convencionales (Ponisio et al., 2015; De Ponti, Rijk e Ittersum, 2012; Thorup, Dresbøll y Kristensen, 2012). Esta limitante es atribuida a la baja disponibilidad de los nutrimentos presentes en los fertilizantes orgánicos, principalmente el N (Cavigelli et al., 2008; Montagu y Goh, 1990). En este trabajo se corroboraron dichos efectos, toda vez que en promedio, los tratamientos orgánicos reflejaron un rendimiento total de 31.6\% menor que el tratamiento inorgánico. Entre los tratamientos orgánicos el T5 fue el que registró el mayor rendimiento total, con una diferencia de $19.4 \%$, comprobando el efecto de la fuente de los fertilizantes orgánicos usados en la producción de frutos, a pesar de que la cantidad de nutrimentos adicionados fue similar entre los tratamientos orgánicos al 100 y $120 \%$. El T5 proporciona un porcentaje de $\mathrm{N}$ a través de la fuente de hidrolizado de proteína de pescado $\left(\mathrm{PHYTAFISH}^{\circledR}\right)$, lo que pudo marcar la diferencia en el rendimiento obtenido entre los tratamientos orgánicos. En este trabajo se observó que el menor rendimiento total obtenido en los tratamientos orgánicos fue debido a la obtención de frutos de menor peso fresco $\mathrm{y}$, a excepción del T5, al menor número y tamaño de fruto por planta, lo que concuerda con lo reportado en otras investigaciones (Bilalis et al., 2018; Ronga et al., 2017), los cuales señalaron que el número de frutos por planta fue influenciado de manera significativa por los diferentes regímenes de fertilización, donde la aplicación de la fertilización inorgánica resultó en un mayor número de frutos por planta con respecto a la fertilización orgánica.

Asimismo, se observa en los últimos racimos evaluados la producción de fruto de algunos tratamientos orgánicos fue similar al del tratamiento inorgánico, lo anterior probablemente se debe a que en esta etapa la disponibilidad de nutrientes en el medio haya aumentado, cubriendo la demanda de las plantas cultivadas con fertilizantes orgánicos, sin embargo, esta mejora en la producción de fruto no fue suficiente para igualar o superar el rendimiento logrado en el tratamiento inorgánico.

El diámetro longitudinal del fruto mostró diferencias estadísticas significativas en el segundo, tercer y cuarto racimo (Cuadro 6). En el segundo y tercer racimo, el tratamiento inorgánico T7 exhibió el mayor diámetro longitudinal, en tanto que en el cuarto racimo los tratamientos orgánicos T1, T5 y T6 presentaron un diámetro longitudinal similar al del tratamiento T7, superando al resto de los tratamientos. Sin embargo, el mayor diámetro longitudinal se observó con el tratamiento inorgánico $\mathrm{T} 7$, superando en $5.8 \%$ a los orgánicos.

El diámetro transversal de fruto fue afectado en todos los racimos, a excepción del primero. En el segundo y tercer racimo el tratamiento inorgánico T7 registró el mayor diámetro transversal de fruto, en tanto que en el cuarto racimo los tratamientos $\mathrm{T} 1$ y $\mathrm{T} 7$ presentaron el mayor valor, siendo similar entre ambos; los tratamientos T1 y T7 exhibieron los valores mayores (2.25 y $2.29 \mathrm{~cm}$, respectivamente) (Cuadro 6).

La firmeza del fruto y los sólidos solubles totales (SST) fueron afectados por los tratamientos en los racimos evaluados. La firmeza fue mayor en los tratamientos orgánicos $\mathrm{T} 1$ y $\mathrm{T} 4$, superando al inorgánico $\mathrm{T} 7$ en 10.3 y $6 \%$, respectivamente. En el primero de los racimos, los tratamientos orgánicos $\mathrm{T} 1$ y T5 superaron la firmeza obtenida con el inorgánico $\mathrm{T} 7$, en tanto que en el segundo racimo los tratamientos orgánicos T1, T3 y T4 y en el tercer racimo los orgánicos T1 y T4 fueron similares al inorgánico T7. En el cuarto racimo, la firmeza de fruto fue mayor en el tratamiento orgánico T1. La variable SST fue mayor en el tratamiento orgánico $\mathrm{T} 4$, superando en $18 \%$ al tratamiento inorgánico T7. Se observó que SST fue mayor en los tratamientos orgánicos en el primer (T1, T3 y T4), segundo (T1, T4 y T6) y tercer racimo (T1, T3. T4, T5 y T6), en tanto que, en el cuarto, los tratamientos orgánicos T2, T3 y T4, fueron similares al inorgánico T7 (Cuadro 7).

De las propiedades del fruto, se ha reportado que, en la producción orgánica, los frutos de tomate contienen cantidades más altas de SST en comparación con tomates cultivados convencionalmente (Bilalis et al., 2018; Rickman-Pieper y Barrett, 2008), pero no todos los estudios han coincidido en este aspecto (Polat, Demir y Erler, 2010; Guajardo et al., 2018). Según los resultados, los frutos de las plantas tratadas orgánicamente obtuvieron $11.20 \%$ más de SST que los frutos con el tratamiento inorgánico. Con excepción del cuarto racimo, se obtuvo menor SST que los tratamientos orgánicos en todos los racimos evaluados a pesar de que con este tratamiento se obtuvo mayor rendimiento (datos no mostrados). Asimismo, se ha 
indicado que el contenido de SST es inversamente proporcional al tamaño de la fruta (Beckles, 2012), lo cual concuerda con el mayor valor de SST en los frutos de menor tamaño obtenido en los tratamientos orgánicos. En este contexto, Preciado-Rangel et al. (2011) y López-Martínez et al. (2016), señalaron que la solución nutritiva Steiner mostró mayor rendimiento y tamaño de fruto, aunque las más altas concentraciones de sólidos solubles totales correspondieron a los tratamientos con solución nutritiva de origen orgánico. La mayor acumulación de sólidos solubles en el fruto pudiera deberse, por una parte, a una menor absorción y acumulación de agua por los frutos, y para superar este problema los frutos acumulan solutos orgánicos como azucares simples (glucosa, fructosa y sacarosa), con lo que se logra disminuir el potencial osmótico, facilitando así la absorción de agua en los frutos (Plaut, Grava, Yehezkel y Matán, 2004; Goykovic y Saavedra, 2007), y por otra parte a la alta concentración de sales particularmente de $\mathrm{Na}$ y $\mathrm{Cl}$ en la solución aplicada (Wu y Kubota, 2008). Por último, se observa una mayor concentración en los frutos obtenidos en el tratamiento $\mathrm{T} 4$, ya que en este se obtuvieron frutos de menor tamaño y peso fresco que el resto de los tratamientos orgánicos. Actualmente, ha crecido la preferencia de los consumidores por alimentos con alta calidad y cultivados con técnicas amigables con el ambiente (Lozano et al., 2018).

Por otra parte, el pH, $\mathrm{CE}, \mathrm{NO}_{3}^{-} \mathrm{y} \mathrm{K}^{+}$en la solución lixiviada mostraron tendencias diferentes, según

Cuadro 6. Efecto de la fertilización orgánica e inorgánica en el diámetro longitudinal y transversal de fruto en tomate uva bajo subirrigación no recirculante.

Table 6. Effect of organic and inorganic fertilization on fruit longitudinal and transverse diameter on grape tomato under nonrecirculating subirrigation.

\begin{tabular}{|c|c|c|c|c|c|}
\hline \multirow{2}{*}{ Tratamientos } & \multicolumn{4}{|c|}{ Racimos } & \multirow{2}{*}{ Promedio } \\
\hline & 1 & 2 & 3 & 4 & \\
\hline \multicolumn{6}{|c|}{ Diámetro longitudinal de fruto $(\mathrm{cm})$} \\
\hline $\mathrm{T} 1$ & $2.61 \mathrm{a}$ & $2.65 \mathrm{~b}$ & $2.71 \mathrm{~b}$ & $2.68 \mathrm{a}$ & $2.66 \mathrm{~b}$ \\
\hline $\mathrm{T} 2$ & $2.60 \mathrm{a}$ & $2.48 \mathrm{~b}$ & $2.53 \mathrm{c}$ & $2.63 \mathrm{bc}$ & $2.56 \mathrm{~cd}$ \\
\hline T3 & $2.64 a$ & $2.66 \mathrm{~b}$ & $2.53 \mathrm{c}$ & $2.59 \mathrm{c}$ & $2.60 \mathrm{bcd}$ \\
\hline $\mathrm{T} 4$ & $2.55 \mathrm{a}$ & $2.47 \mathrm{~b}$ & $2.54 \mathrm{c}$ & $2.60 \mathrm{c}$ & $2.54 \mathrm{~d}$ \\
\hline T5 & $2.70 \mathrm{a}$ & $2.53 b$ & $2.62 \mathrm{bc}$ & $2.68 \mathrm{a}$ & $2.63 \mathrm{bc}$ \\
\hline T6 & $2.64 \mathrm{a}$ & $2.59 \mathrm{~b}$ & $2.53 \mathrm{c}$ & $2.68 \mathrm{a}$ & $2.61 \mathrm{bcd}$ \\
\hline $\mathrm{T} 7$ & $2.60 \mathrm{a}$ & $2.91 \mathrm{a}$ & $2.86 \mathrm{a}$ & $2.67 \mathrm{ab}$ & $2.76 \mathrm{a}$ \\
\hline ANOVA $P \leq$ & 0.936 & 0.003 & 0.001 & 0.002 & 0.001 \\
\hline \multicolumn{6}{|c|}{ Diámetro transversal de fruto $(\mathrm{cm})$} \\
\hline $\mathrm{T} 1$ & $2.18 \mathrm{a}$ & $2.22 \mathrm{~b}$ & $2.28 \mathrm{~b}$ & $2.35 \mathrm{a}$ & $2.25 \mathrm{ab}$ \\
\hline $\mathrm{T} 2$ & $2.20 \mathrm{a}$ & $2.22 \mathrm{~b}$ & $2.18 \mathrm{c}$ & $2.28 b c$ & $2.22 \mathrm{bc}$ \\
\hline T3 & $2.26 \mathrm{a}$ & $2.19 b c$ & $2.18 \mathrm{c}$ & $2.23 \mathrm{c}$ & $2.22 \mathrm{bc}$ \\
\hline $\mathrm{T} 4$ & $2.19 \mathrm{a}$ & $2.02 \mathrm{~d}$ & $2.21 \mathrm{c}$ & $2.25 b c$ & $2.17 \mathrm{c}$ \\
\hline T5 & $2.19 \mathrm{a}$ & $2.11 \mathrm{~cd}$ & $2.18 \mathrm{c}$ & $2.25 \mathrm{bc}$ & $2.19 b c$ \\
\hline T6 & $2.23 \mathrm{a}$ & $2.14 \mathrm{bc}$ & $2.18 \mathrm{c}$ & $2.25 \mathrm{bc}$ & $2.20 \mathrm{bc}$ \\
\hline $\mathrm{T} 7$ & $2.15 \mathrm{a}$ & $2.33 \mathrm{a}$ & $2.41 \mathrm{a}$ & $2.30 \mathrm{ab}$ & $2.29 \mathrm{a}$ \\
\hline ANOVA $P \leq$ & 0.912 & 0.001 & 0.001 & 0.007 & 0.011 \\
\hline
\end{tabular}

Medias en la misma columna con la misma letra no presentan diferencias significativas, según la prueba DMS $(P \leq 0.05)$. ANOVA $=$ análisis de varianza. Means in the same column with the same letter do not show significant differences, according to the LSD test $(P \leq 0.05)$. ANOVA= analysis of variance. 
la fertilización inorgánica y orgánica (Figura 1). El pH del lixiviado se incrementó a lo largo del experimento en todos los tratamientos. A los 120 y 150 DDT el tratamiento inorgánico $\mathrm{T} 7$ presentó un $\mathrm{pH}$ mayor que los orgánicos, en tanto que los orgánicos T2 y T5 presentaron un menor $\mathrm{pH}$ durante el estudio (Figura 1A). La CE menor se registró en el tratamiento inorgánico T7. La CE del lixiviado de los orgánicos aumentó de los 5 a 120 DDT, entre ellos, T3 y T4 presentaron una $\mathrm{CE}$ menor en el lixiviado, en tanto que la del lixiviado del inorgánico T7 aumentó de los 5 a 30 DDT, observándose constante posteriormente (Figura 1B). La concentración de $\mathrm{NO}_{3}^{-}$del lixiviado de los tratamientos orgánicos se mantuvo constante de los 5 a 90 DDT aumentando durante los 120 y 150 DDT, en tanto que en el inorgánico T7 se incrementó a los 30, 120 y 150 DDT (Figura 1C). La concentración de $\mathrm{K}^{+}$en el lixiviado fue mayor en los orgánicos, incrementando conforme trascurrieron los DDT con una mayor concentración en el T4, en tanto que el inorgánico T7 presentó una concentración de $\mathrm{K}^{+}$estable durante el periodo evaluado (Figura 1D).

Por otra parte, el $\mathrm{pH}$ del lixiviado se incrementó en los tratamientos conforme avanzó los DDT, con un mayor incremento en el tratamiento inorgánico T7 al final del experimento alcanzando un $\mathrm{pH}$ de 7.10, mientras que, en promedio, los tratamientos orgánicos alcanzaron un $\mathrm{pH}$ de 6.22; sin embargo, el mayor $\mathrm{pH}$ del tratamiento inorgánico T7 no pareció afectar el crecimiento y rendimiento de las plantas de tomate.

Cuadro 7. Efecto de la fertilización orgánica e inorgánica en la firmeza y sólidos solubles totales de fruto en tomate uva bajo subirrigación no recirculante.

Table 7. Effect of organic and inorganic fertilization on fruit firmness and total soluble solids of fruit on grape tomato under nonrecirculating subirrigation.

\begin{tabular}{|c|c|c|c|c|c|}
\hline \multirow{2}{*}{ Tratamientos } & \multicolumn{4}{|c|}{ Racimos } & \multirow{2}{*}{ Promedic } \\
\hline & 1 & 2 & 3 & 4 & \\
\hline \multicolumn{6}{|c|}{ Firmeza (N-newtons) } \\
\hline $\mathrm{T} 1$ & $9.18 \mathrm{a}$ & 7.33abc & $6.60 \mathrm{abc}$ & $8.35 \mathrm{a}$ & $7.87 \mathrm{a}$ \\
\hline $\mathrm{T} 2$ & $6.05 \mathrm{c}$ & $6.22 \mathrm{c}$ & $6.04 \mathrm{bc}$ & $5.53 \mathrm{~cd}$ & $5.96 \mathrm{e}$ \\
\hline $\mathrm{T} 3$ & $6.36 \mathrm{c}$ & $8.32 \mathrm{a}$ & $4.75 \mathrm{~d}$ & $5.80 \mathrm{bcd}$ & $6.31 \mathrm{de}$ \\
\hline $\mathrm{T} 4$ & $8.11 \mathrm{~b}$ & $8.62 \mathrm{a}$ & $6.74 \mathrm{ab}$ & $6.56 \mathrm{~b}$ & $7.51 \mathrm{ab}$ \\
\hline $\mathrm{T} 5$ & $8.42 \mathrm{ab}$ & $6.78 \mathrm{bc}$ & $5.78 \mathrm{c}$ & $6.11 \mathrm{bc}$ & $6.77 \mathrm{~cd}$ \\
\hline T6 & $6.17 \mathrm{c}$ & $5.95 \mathrm{c}$ & $5.70 \mathrm{c}$ & $6.44 \mathrm{~b}$ & $6.06 \mathrm{e}$ \\
\hline $\mathrm{T} 7$ & $7.82 \mathrm{~b}$ & $8.12 \mathrm{ab}$ & $7.02 \mathrm{a}$ & $5.28 \mathrm{~d}$ & $7.06 \mathrm{bc}$ \\
\hline ANOVA $P \leq$ & 0.001 & 0.004 & 0.001 & 0.001 & 0.001 \\
\hline \multicolumn{6}{|c|}{ Solidos solubles totales $\left({ }^{\circ}\right.$ Brix $)$} \\
\hline $\mathrm{T} 1$ & $8.83 \mathrm{ab}$ & $9.05 \mathrm{ab}$ & $8.82 \mathrm{a}$ & $7.88 \mathrm{c}$ & $8.65 b$ \\
\hline $\mathrm{T} 2$ & $7.68 \mathrm{~cd}$ & $8.20 \mathrm{bc}$ & $7.86 \mathrm{bc}$ & $8.44 a b$ & $8.05 \mathrm{bc}$ \\
\hline $\mathrm{T} 3$ & $8.55 \mathrm{abc}$ & $8.43 b$ & $8.93 \mathrm{a}$ & $8.57 \mathrm{a}$ & $8.62 \mathrm{~b}$ \\
\hline $\mathrm{T} 4$ & $9.50 \mathrm{a}$ & $9.88 \mathrm{a}$ & $9.04 \mathrm{a}$ & $8.76 \mathrm{a}$ & $9.29 \mathrm{a}$ \\
\hline T5 & $8.28 \mathrm{bc}$ & $8.37 \mathrm{bc}$ & $8.64 \mathrm{ab}$ & $8.01 \mathrm{bc}$ & $8.33 b$ \\
\hline T6 & $8.24 \mathrm{bc}$ & $8.84 \mathrm{ab}$ & $9.44 \mathrm{a}$ & $7.37 \mathrm{~d}$ & $8.48 b$ \\
\hline $\mathrm{T} 7$ & $7.11 \mathrm{~d}$ & $7.30 \mathrm{c}$ & $7.55 \mathrm{c}$ & $8.46 \mathrm{a}$ & $7.61 \mathrm{c}$ \\
\hline ANOVA $P \leq$ & 0.002 & 0.006 & 0.006 & 0.001 & 0.001 \\
\hline
\end{tabular}

Medias en la misma columna con la misma letra no presentan diferencias significativas, según la prueba DMS $(P \leq 0.05)$. ANOVA $=$ análisis de varianza. Means in the same column with the same letter do not show significant differences, according to the LSD test $(P \leq 0.05)$. ANOVA= analysis of variance. 


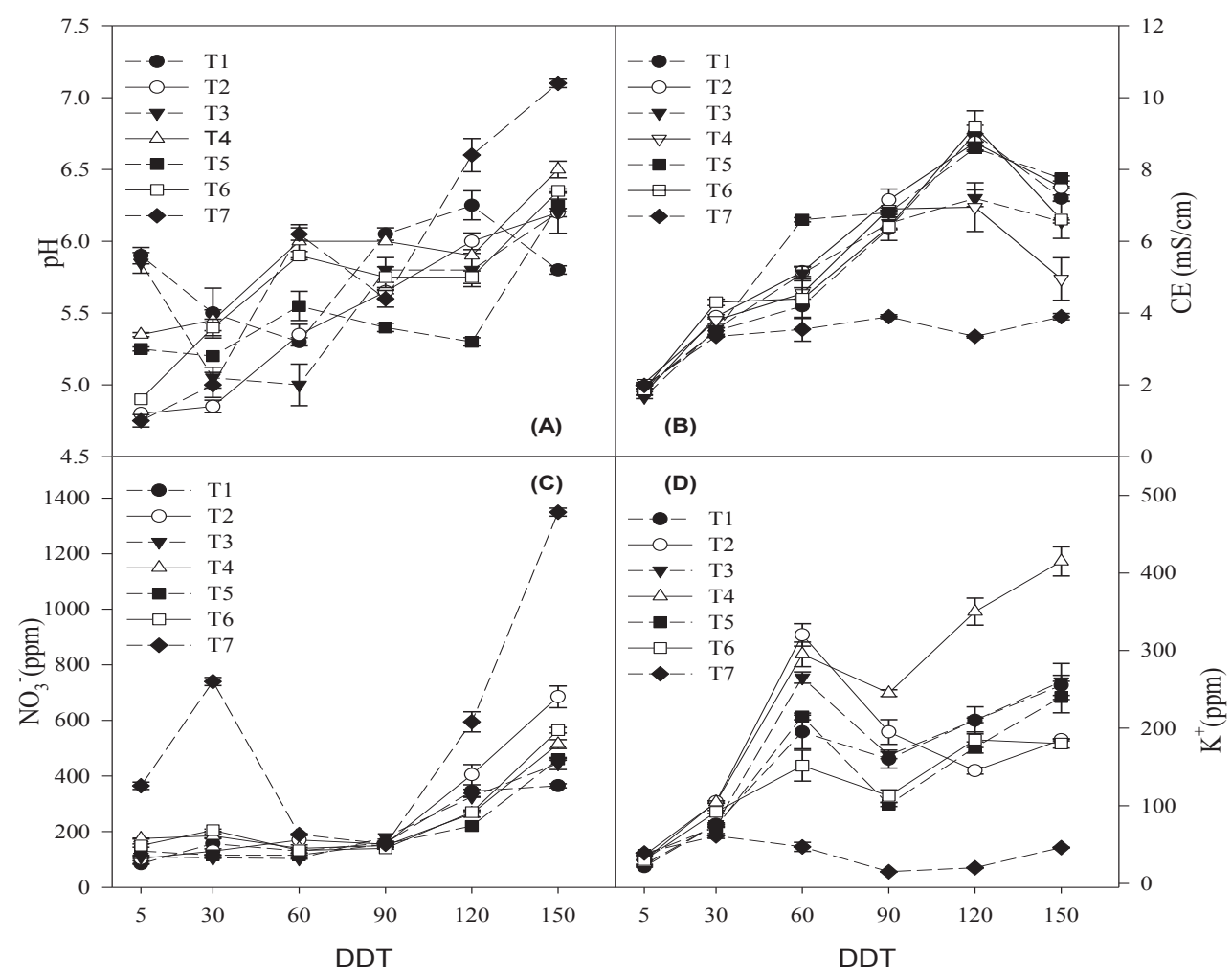

Figura 1. Tendencia de la concentración de $\mathrm{NO}_{3}^{-}, \mathrm{K}^{+}, \mathrm{pH}$ y $\mathrm{CE}$ en el lixiviado en función del tipo de fertilización aplicada (orgánica e inorgánica). Las barras representan la desviación estándar de la media.

Figure 1. Trend of $\mathrm{NO}_{3}^{-}, \mathrm{K}^{+}, \mathrm{pH}$ y $\mathrm{CE}$ concentration in leachate according to the type of fertilization applied (organic and inorganic). Bars represent the standard deviation of the mean.

Por otro lado, la CE es un indicador indirecto de la concentración de sales en una solución (Yan et al., 2011). En la fertilización inorgánica los nutrimentos están disponibles inmediatamente en su forma iónica en el medio, en tanto que en la fertilización orgánica los nutrimentos deben pasar por el proceso de mineralización para estar disponibles en forma iónica (Bergstrand et al., 2018). Lo anterior implica que la CE sería mayor en el tratamiento control conforme transcurrieron los DDT, según lo ha reportado Rippy et al. (2004), sin embargo, en este trabajo la CE fue menor en el tratamiento inorgánico y se mantuvo constante después de los 30 DDT, alcanzando un máximo a los $150 \mathrm{DDT}$ de $3.90 \mathrm{mS} \mathrm{cm} \mathrm{cm}^{-1}$; mientras que en los tratamientos orgánicos la $\mathrm{CE}$ tendió a incrementar conforme pasaron los DDT, con un máximo a los $120 \mathrm{DDT}\left(8.28 \mathrm{mS} \mathrm{cm} \mathrm{cm}^{-1}\right)$. Lo anterior puede obedecer a que al aplicar los nutrientes en forma iónica (tratamiento inorgánico), existió una mayor absorción, reduciendo la cantidad de sales del medio, mientras que en los tratamientos orgánicos los iones al tardar más tiempo en liberarse provocaron el incremento de la CE. Por otro lado, Bergstrand et al. (2018) reportaron que la concentración de $\mathrm{NO}_{3}{ }^{-}$en el medio de crecimiento con fertilización orgánica se mantuvo baja en las primeras semanas, aumentando después de 3 a 5 semanas de establecido el cultivo de Pelargonium. En esta investigación se observó una tendencia similar en cuanto a la concentración de $\mathrm{NO}_{3}{ }^{-}$en el lixiviado, con una concentración bajo y constante hasta los 90 DDT en todos los tratamientos orgánicos respecto al tratamiento inorgánico. La baja concentración de $\mathrm{NO}_{3}^{-}$en el lixiviado de los tratamientos orgánicos durante los 90 DDT, pareció obedecer a que la tasa de absorción de la planta fue superior a la mineralización, reduciendo su concentración en el medio de crecimiento $\mathrm{y}$, por lo tanto, en el lixiviado. 
Se observó que los atributos morfológicos de fruto: perímetro, área, anchura a media altura, anchura máxima, altura a media anchura, altura máxima, área y grosor de pericarpio fueron afectados por los tratamientos. Por su parte, el índice de forma del fruto (I y II) no mostró efectos significativos entre los tratamientos evaluados (Cuadro 8). Los descriptores morfológicos de fruto que se evaluaron, el perímetro, área, anchura a media altura y anchura máxima, exhibieron valores mayores en plantas fertilizadas con la solución inorgánica (T7). Por otra parte, para las variables altura a media anchura y altura máxima de fruto, los tratamientos orgánicos T3, T5, T6 y el tratamiento convencional T7 mostraron valores estadísticamente similares. Los descriptores relacionados a la sección latitudinal de fruto, área y grosor de pericarpio, fueron mayores en plantas fertilizadas con la solución inorgánica (T7). La descripción de cada atributo morfológico de fruto se muestra en la Cuadro 8.
En cuanto a los atributos morfológicos de fruto en este trabajo, aun cuando el tratamiento control presentó los valores mayores, se observó que algunos tratamientos orgánicos presentaron un comportamiento similar, principalmente los tratamientos T3, T5 y T6, ratificando el mejor comportamiento de estos tratamientos T5 y T7 en los parámetros de producción y calidad de fruto. Dicha similitud en los efectos de la fertilización orgánica e inorgánica en los caracteres morfológicos de los frutos de tomate uva ha sido reportado por Lozano et al. (2018), principalmente en los componentes de las mediciones básicas. Dichos autores al someter plantas de tomate uva a nutrición orgánica e inorgánica, no observaron diferencias en los componentes del índice de forma de fruto, lo cual se observó en este trabajo; sin embargo, para estos descriptores los tratamientos orgánicos e inorgánicos mostraron valores mayores que 1 , que indican frutas alargadas.

Cuadro 8. Efecto de la fertilización orgánica e inorgánica sobre los atributos morfológicos de fruto en tomate uva bajo subirrigación no recirculante.

Table 8. Effect of organic and inorganic fertilization on the morphological attributes of grape tomato under non-recirculating subirrigation.

\begin{tabular}{|c|c|c|c|c|c|c|c|c|}
\hline Descriptores morfológicos & $\mathrm{T} 1$ & $\mathrm{~T} 2$ & $\mathrm{~T} 3$ & $\mathrm{~T} 4$ & $\mathrm{~T} 5$ & T6 & $\mathrm{T} 7$ & ANOVA $P \leq$ \\
\hline \multicolumn{9}{|c|}{ Medidas básicas } \\
\hline Perímetro $(\mathrm{cm})$ & $9.12 b c$ & $9.09 \mathrm{c}$ & $9.51 b c$ & $9.29 b c$ & $9.54 b$ & $9.41 b c$ & $10.00 \mathrm{a}$ & 0.001 \\
\hline Área $\left(\mathrm{cm}^{2}\right)$ & $5.72 \mathrm{c}$ & $5.64 \mathrm{c}$ & $6.14 b c$ & $5.87 \mathrm{bc}$ & $6.25 b$ & $6.05 \mathrm{bc}$ & $6.85 \mathrm{a}$ & 0.001 \\
\hline Anchura a media altura $(\mathrm{cm})$ & $2.36 \mathrm{bc}$ & $2.31 \mathrm{c}$ & $2.41 b$ & $2.39 \mathrm{bc}$ & $2.45 b$ & $2.42 b$ & $2.59 \mathrm{a}$ & 0.001 \\
\hline Anchura máxima (cm) & $2.38 \mathrm{bc}$ & $2.34 \mathrm{c}$ & $2.45 b$ & $2.42 b c$ & $2.48 b$ & $2.44 b$ & $2.64 \mathrm{a}$ & 0.001 \\
\hline Altura a media anchura $(\mathrm{cm})$ & $2.95 \mathrm{c}$ & $2.95 b$ & $3.08 \mathrm{ab}$ & $2.96 \mathrm{~b}$ & $3.08 \mathrm{ab}$ & $3.05 \mathrm{ab}$ & $3.20 \mathrm{a}$ & 0.038 \\
\hline Altura máxima (cm) & $2.98 b$ & $2.99 \mathrm{c}$ & $3.14 \mathrm{ab}$ & $3.00 \mathrm{~b}$ & $3.13 \mathrm{ab}$ & $3.10 \mathrm{ab}$ & $3.25 \mathrm{a}$ & 0.024 \\
\hline \multicolumn{9}{|c|}{ Índice de forma de fruto } \\
\hline Índice de forma de fruto (I) & $1.25 \mathrm{a}$ & $1.27 \mathrm{a}$ & $1.28 \mathrm{a}$ & $1.24 \mathrm{a}$ & $1.26 \mathrm{a}$ & $1.27 \mathrm{a}$ & $1.23 \mathrm{a}$ & 0.379 \\
\hline Índice de forma de fruto (II) & $1.25 \mathrm{a}$ & $1.27 \mathrm{a}$ & $1.28 \mathrm{a}$ & $1.24 \mathrm{a}$ & $1.25 \mathrm{a}$ & $1.26 \mathrm{a}$ & $1.23 \mathrm{a}$ & 0.555 \\
\hline \multicolumn{9}{|c|}{ Sección latitudinal } \\
\hline Área de pericarpio & $2.54 \mathrm{c}$ & $2.51 \mathrm{c}$ & $2.73 b c$ & $2.61 b c$ & $2.78 \mathrm{~b}$ & $2.69 b c$ & $3.05 \mathrm{a}$ & 0.001 \\
\hline Grosor de pericarpio & $0.3125 \mathrm{c}$ & $0.3092 \mathrm{c}$ & $0.3205 b c$ & $0.3147 b c$ & $0.3262 b$ & $0.3205 b c$ & $0.3420 \mathrm{a}$ & 0.001 \\
\hline
\end{tabular}

Medias en la misma columna con la misma letra no presentan diferencias significativas, según la prueba DMS $(P \leq 0.05)$. ANOVA= análisis de varianza. Means in the same column with the same letter do not show significant differences, according to the LSD test $(P \leq 0.05)$. ANOVA= analysis of variance. 


\section{CONCLUSIONES}

La nutrición orgánica no aumentó el rendimiento de tomate uva con respecto a la nutrición convencional; sin embargo, contribuyó a obtener frutos de mayor calidad, así como un crecimiento y atributos morfológicos similares a la nutrición inorgánica. Se confirma que la dosis y la fuente de la nutrición orgánica influyeron en el comportamiento del cultivo, con una mejor respuesta al emplear como dosis de $\mathrm{N}$ el hidrolizado de proteína de pescado al $100 \%$.

\section{DECLARACIÓN DE ÉTICA}

No aplicable.

\section{CONSENTIMIENTO PARA PUBLICACIÓN}

No aplicable.

\section{DISPONIBILIDAD DE DATOS}

Los conjuntos de datos utilizados o analizados durante el estudio actual están disponibles del autor correspondiente a solicitud razonable.

\section{CONFLICTO DE INTERESES}

Los autores declaran que no tienen intereses en competencia.

\section{FONDOS}

La investigación fue financiada con fondos internos institucionales de la Universidad Autónoma Agraria Antonio Narro.

\section{CONTRIBUCIÓN DE LOS AUTORES}

Conceptualización:C.J.L.C.yJ.A.G.F.Metodología: C.J.L.C., J.A.G.F. y P.P.R. Software: A.R.D., C.J.L.C. y E.T.D. Investigación: C.J.L.C., P.P.R., J.A.G.F. Análisis formal de datos: E.T.D., J.C.R.O. y A.R.D. Curación de datos: J.C.R.O. Preparación del borrador original: C.J.L.C., J.A.G.F. y J.C.R.O. Revisión y edición: P.P.R., E.T.D. y C.J.L.C. Administración del proyecto: C.J.L.C. Adquisición de fondos: C.J.L.C. Todos los autores leyeron y aprobaron el manuscrito final.

\section{LITERATURA CITADA}

Agehara, S., \& Warncke, D. D. (2005). Soil moisture and temperature effects on nitrogen release from organic nitrogen sources. Soil Science Society America Journal, 69, 1844-1855. https://doi.org/10.2136/sssaj2004.0361

Beckles, D. M. (2012). Factors affecting the postharvest soluble solids and sugar content of tomato (Solanum lycopersicum L.) fruit. Postharvest Biology and Technology, 63(1), 129-140. https://doi.org/10.1016/j.postharvbio.2011.05.016

Beman, J. M., Arrigo, K. R., \& Matson, P. A. (2005). Agricultural runoff fuels large phytoplankton blooms in vulnerable areas of the ocean. Nature, 434, 211-214. doi: https://doi.org/10.1038/ nature 03370

Bergstrand, K. J., Löfkvist, K., \& Asp, H. (2018). Dynamics of nitrogen availability in pot grown crops with organic fertilization. Biological Agriculture \& Horticulture, 35, 1-8. https://doi.org/10.1080/01448765.2018.1498389

Bi, G., Evans, W. B., Spiers, J. M., \& Witcher, A. L. (2010). Effects of organic and inorganic fertilizers on marigold growth and flowering. Hortscience, 45(9), 1373-1377. https://doi. org/10.21273/HORTSCI.45.9.1373

Bilalis, D., Krokida, M., Roussis, I., Papastylianou, P., Travlos, I., Cheimona, N., \& Dede, A. (2018). Effects of organic and inorganic fertilization on yield and quality of processing tomato (Lycopersicon esculentum Mill.). Folia Horticulturae, 30(2), 321-332. https://doi.org/10.2478/fhort-2018-0027

Cavigelli, M. A., Teasdale, J. R., \& Conklin, A. E. (2008). Longterm agronomic performance of organic and conventional field crops in the mid-Atlantic region. Agronomy Journal, 100(3), 785-794. https://doi.org/10.2134/agronj2006.0373

Colla, G., Rouphael, Y., Canaguier, R., Svecova, E., \& Cardarelli, M. (2014). Biostimulant action of a plant-derived protein hydrolysate produced through enzymatic hydrolysis. Frontiers in Plant Science, 5(448), 1-6. https://doi.org/10.3389/ fpls.2014.00448

Colla, G., \& Rouphael, Y. (2015). Biostimulants in horticulture. Scientia Horticulturae, 196, 39-48. https://doi.org/10.1016/j. scienta.2015.10.044

Cristiano, G., Pallozzi, E., Conversa, G., Tufarelli, V., \& Lucia, B. (2018). Effects of an animal-derived biostimulant on the growth and physiological parameters of potted snapdragon (Antirrhinum majus L.). Frontiers in Plant Science, 9. https:// doi.org/10.3389/fpls.2018.00861

De Ponti, T., Rijk, B., \& Ittersum, M. K. (2012). The crop yield gap between organic and conventional agriculture. Agricultural Systems, 108, 1-9. https://doi.org/10.1016/j.agsy.2011.12.004

Ertani, A., Schiavon, M., Muscolo, A., \& Nardi, S. (2013). Alfalfa plant-derived biostimulant stimulate short-term growth of salt stressed Zea mays L. plants. Plant and soil, 364(1-2), 145-158. https://doi.org/10.1007/s11104-012-1335-Z

Gaskell, M., \& Smith, R. (2007). Nitrogen sources for organic vegetable crops. HortTechnology, 17(4), 431-441. https://doi. org/10.21273/HORTTECH.17.4.431

Gonzalo, M. J., \& Van der Knaap, E. (2008). A comparative analysis into the genetic bases of morphology in tomato varieties exhibiting elongated fruit shape. Theoretical and Applied Genetics 116, 647-656. https://doi.org/10.1007/s00122-0070698-7 
Goykovic C., V., \& Saavedra del R., G. (2007). Algunos efectos de la salinidad en el cultivo de tomate y prácticas agronómicas de su manejo. IDESIA, 25(3), 47-58. https://doi.org/10.4067/ S0718-34292007000300006

Guajardo-Ríos, O., Lozano-Cavazos, C. J., Valdez-Aguilar, L. A., Benavides-Mendoza, A., Ibarra-Jiménez, L., AscacioValdés, J. A., \& Aguilar- González, C. N. (2018). Animalbased organic nutrition can substitute inorganic fertigation in soilless-grown grape tomato. Acta Agriculturae Scandinavica Section B-Soil \& Plant Science, 68(1), 77-85. https://doi.org/1 0.1080/09064710.2017.1367030

Haley, T. B., \& Reed, D. W. (2004). Optimum potassium concentrations in recirculating subirrigation for selected greenhouse crops. HortScience, 39(6), 1441-1444. https://doi. org/10.21273/HORTSCI.39.6.1441

Huang, J., Pray, C., \& Rozelle, S. (2002). Enhancing the crops to feed the poor. Nature, 418, 678-684. https://doi.org/10.1038/ nature 01015

Khandaker, M. M., Rohani, F., Dalorima, T., \& Mat, N. (2017). Effects of different organic fertilizers on growth, yield and quality of Capsicum annuum L. Var. Kulai (Red Chilli Kulai). Biosciences Biotechnology Research Asia, 14(1), 185-192. https://doi.org/10.13005/bbra/2434.

López-Martínez, J. D., D. A. Vázquez-Díaz, J. R. Esparza-Rivera, J. L. García-Hernández, Castruita-Segura, M. A., \& PreciadoRangel, P. (2016). Rendimiento y calidad nutracéutica de frutos de tomate producidos con soluciones nutritivas preparadas con materiales orgánicos. Revista Fitotecnia Mexicana, 39(4), 409-414.

Lozano- Cavazos, C. J., Valdez-Aguilar, L. A., Ibarra-Jiménez, L., Ascacio-Valdés, J. A., Benavides-Mendoza, A., AguilarGonzález, C. N., \& Guajardo-Ríos, O. (2018). Animal-based organic nutrition induces comparable fruit quality to that of inorganic fertigation in soilless-grown grape tomato. Acta Agriculturae Scandinavica, Section B-Soil \& Plant Science, 68(6), 515-523. https://doi.org/10.1080/09064710.2018.1437215

Lucini, L., Rouphael, Y., Cardarelli, M., Canguier, R., Kumar, P., \& Colla, G. (2015). The effect of a plant-derived biostimulant on metabolic profiling and crop performance of lettuce grown under saline conditions. Scientia Horticulturae, 182(23), 124133. https://doi.org/10.1016/j.scienta.2014.11.022

Montagu, K. D., \& Goh, K. M. (1990). Effects of forms and rates of organic and inorganic nitrogen fertilisers on the yield and some quality indices of tomatoes (Lycopersicon esculentum Miller). New Zealand Journal of Crop and Horticultural Science, 18(1), 31-37. https://doi.org/10.1080/01140671.199 0.10428067

Muller, A., Schader, C., El-Hage Scialabba, N., Brüggemann, J., Isensee, A., Erb, K.,... Niggli, U. (2017). Strategies for feeding the world more sustainably with organic agriculture. Nature Communications, 8, 1290. https://doi.org/10.1038/ s41467-017-01410-w.

Plaut, Z., Grava, A., Yehezkel, Ch., \& Matán, E. (2004). How do salinity and water stress affect transport of water assimilates and ions to tomato fruits? Physiologia Plantarum, 122(4), 429-442. https://doi.org/10.1111/j.1399-3054.2004.00416.x

Polat, E., Demir, H., \& Erler, F. (2010). Yield and quality criteria in organically and conventionally grown tomatoes in Turkey. Scientia Agricola, 67(4), 424-429. https://doi.org/10.1590/ S0103-90162010000400008
Ponisio, L. C., M'Gonigle, L. K., Mace, K. C., Palomino, J., Valpine, P., \& Kremen, C. (2015). Diversification practices reduce organic to conventional yield gap. Proceedings of the Royal Society B, 282, 20141396. https://doi.org/10.1098/ rspb.2014.1396

Preciado-Rangel, P., Fortis-Hernández, M., García-Hernández, J. L., Rueda Puente, E. O., Esparza Rivera, J. R., Lara Herrera, A.,... Orozco Vidal, J. A. (2011). Evaluación de soluciones nutritivas orgánicas en la producción de tomate en invernadero. Interciencia, 36(9), 689-693.

Rickman-Pieper, J., \& Barrett, D. M. (2008). Effects of organic and conventional production systems on quality and nutritional parameters of processing tomatoes. Journal of the Science Food Agriculture, 89, 177-194. https://doi.org/10.1002/jsfa.3437

Rippy, J. F. M., Peet, M. M., Louws, F. J., Nelson, P. V., Orr, D. B., \& Sorensen, K. A. (2004). Plant development and harvest yields of greenhouse tomatoes in six organic growing systems. HortScience, 39(2), 223-229. https://doi.org/10.21273/ HORTSCI.39.2.223

Rodríguez, G. R., Moyseenko, J. B., Robbins, M. D., HuarachiMorejón, N., Francis, D. M., \& Knaap, E. van der. (2010). Tomato analyzer: A useful software application to collect accurate and detailed morphological and colorimetric data from two-dimensional objects. Journal of Visualized Experiments, 37, e1856. https://doi.org/10.3791/1856

Ronga, D., Zaccardelli, M., Lovelli, S., Perrone, D., Francia, E., Milc, J., Ulrici, A., \& Pecchioni, N. (2017). Biomass production and dry matter partitioning of processing tomato under organic vs. conventional cropping systems in a Mediterranean environment. Science Horticulturae, 224, 163-170. https://doi. org/10.1016/j.scienta.2017.05.037

Santi, C., Zamboni, A., Varanini, Z., \& Pandolfini, T. (2017). Growth stimulatory effects and genome-wide transcriptional changes produced by protein hydrolysates in maize seedlings. Frontiers Plant Science 8, 433. https://doi.org/10.3389/ fpls.2017.00433

Stadler, C., Tucher, S. von, Schmidhalter, U., Gutser, R., \& Heuwinkel, H. (2006). Nitrogen release from plant-derived and industrially processed organic fertilizers used in organic horticulture. Journal of Plant Nutrition and Soil Science, 169 , 549-556. https://doi.org/10.1002/jpln.200520579

Steiner, A. A. (1984). The universal nutrient solution. In Proceedings of the 6th International Congress on Soilless Culture. (pp. 633650). Lunteren, The Netherlands: ISOSC.

Thorup-Kristensen, K., Dresbøll, D. B., \& Kristensen, H. L. (2012). Crop yield root growth, and nutrient dynamics in a conventional and three organic cropping systems with different levels of external inputs and $\mathrm{N}$ re-cycling through fertility building crops. European Journal of Agronomy, 37(1), 66-82. https://doi.org/10.1016/j.eja.2011.11.004

Tuomisto, H. L., Hodge, I. D., Riordan, P., \& Macdonald, D. W. (2012). Does organic farming reduce environmental impacts? -A meta-analysis of European research. Journal of Environmental Management, 112, 309-320. https://doi. org/10.1016/j.jenvman.2012.08.018

Yan, W., Zheng-xiang, W., Xiaojuan, L., Hui, X., Liyan, W., \& Hong-da, H. (2011). Measurement of soil electric conductivity and relationship between soluble salt content and electrical conductivity in Tianjin Coastal Area. Tianjin Agricultural Sciences, 17, 18-21. 
Wu, M., \& Kubota, Ch. (2008). Effects of high electrical conductivity of nutrient solution and its application timing on lycopene, chlorophyll and sugar concentrations of hydroponic tomatoes during ripening. Scientia Horticulturae, 116(2), 122129. https://doi.org/10.1016/j.scienta.2007.11.014
Xu, C., \& Mou, B. (2017). Drench application of fish-derived protein hydrolysates affects lettuce growth, chlorophyll content, and gas exchange. Hort Technology, 27(4), 539-543. https://doi.org/10.21273/HORTTECH03723-17 